\title{
EVOLUTION DANS LE TEMPS DES PEUPLEMENTS DES SABLES ENVASES EN BAIE DF CONCARNEAU (BRETAGNE)
}

\author{
Pierre Chardy et Michel Glémarec \\ Centre Océanologique de Bretagne - 29273 Brest Ciédex \\ Universaté de Bretagne Occidentale - Laboratoure d'Océanographue \\ Biologique - 29283 Brest Cédex - France
}

\section{ABSTRACT}

- Qualitative and quantitative studies were carried out at three localities at different times of the year, from 1970 to 1974. Multivariate analysis, using inertia techniques, elucidated the major features of the principal sources of variation in time and space. The first main variation, in spite of the similarity of edaphic factors, was related to the spatial distribution of the samples among the stations. Replicate sampling procedures facilitated identification of the heterogeneous components of the environment. To estimate the importance of the time fluctuations at each of the three stations, an attempt was made to locate them along a climatic gradient. Time evolution is governed by two phenomena: annual cycle and climactic trend. Species diversity analysis gives an interpretation of this evolution.-

\section{INTRODUCTION}

En baie de Concarneau, les entités de peuplement sont bien connues, ainsi que les facteurs écologiques qui les gouvernent. Le présent travail a pour but de rechercher une éventuelle synchronisation des fluctuations dans le temps des peuplements caractéristiques des sables envasés de la baie de Concarneau.

L'analyse s'effectue en deux temps :

- étude globale des facteurs "temps" et "espace" sur la composition qualitative et quantitative des peuplements. Importance relative de ces deux sources de variations;

- étude particulière du facteur temps : mise en évidence des variations saisonnières et pluriannuelles, communes aux trois stations (principal objectif de l'étude).

\section{MATERIEL ET METHODES}

\section{Récolte des données}

Durant cinq années, de 1970 à 1974, 3 stations ont été régulièrement échantillonnées, 4 fois par an à intervalles réguliers, à raison de 4 à 8 bennes Smith-Mac Intyre par station; ce qui représente 331 prélèvements. Les trois stations appartiennent à la même unité édaphique, les sables envasés définis par le type de peuplement à "Amphiura." Elles s'étagent le long du gradient climatique (Glémarec, 1973).

Station Beg-Meil : 10 mètres, étage infralittoral, à l'abri des houles dominantes, communauté à Acrocnida brachiata - Clymene oerstedii.

Station Baie de Concarneau : 28 mètres, étage circalittoral côtier, hydrodynamiquement stable, cummunautè à Amphiura filiformis - Lumbrinereis gracilis. 
Station Mousterlin : 18 mètres, station intermédiaire entre l'infralittoral et le circalittoral côtier. Soumise aux houles de sud-est fréquentes. Peuplement également intermédiaire.

Entre ces 3 stations, l'analyse granulométrique globale ne montre pas de variation significative du taux de pélites. Ce paramètre mesuré au niveau du premier centimètre de surface, permet d'appréhender le caractère de stabilité de la couche superficielle : Boucher (1975), montre ainsi que la station de Beg-Meil est plus stable et légèrement plus envasée que les deux autres. Les 23 espèces les mieux représentées sont retenues pour l'analyse.

\section{METHODE D'ANALYSE DES DONNEES}

L'outil mathématique utilisé est 1 'analyse d'inertie, permettant de visualiser globalement les ressemblances faunistiques entre prélèvements ou groupes de prélèvements caractérisés par une date et une position dans l'espace. Une analyse des diverses variantes possibles a déjà été proposée par chardy, Glémarec \& Laured (1976). Cependant, il est utile de préciser la signification des options retenues pour cette étude.

\section{Etude globale des variations spatio-temporelles}

Afin de dégager une structure qui ne soit pas uniquement due aux fluctuations des espèces à forte densité (telle qu'Amphiura filifformis), les effectifs des espèces sont réduits et centrés. Le centrage a la propriété de faire disparaître le décalage systématique de densité entre deux espèces. L'intérêt de 1 'analyse consiste à prendre en compte les espèces dont les effectifs varient de la même façon ; les différences d'abondance importent peu. La réduction permet de pondérer le rôle des espèces dont la densité peu fluctuer largement d'un prëlèvement à l'autre. Il faut noter par ailleurs, que ces options permettent de prendre en compte les différences de densité entre prélèvements. Afin d'êliminer les biais dus aux phénomènes de surdispersion (particulièrement sensibles dans le cas de l'échantillonnage par benne) les données ont subies une transformation logarithmique. La distance retenue est une distance euclidienne, qui nous ramène au cas de l'analyse d'inertie d'une matrice de corrélation entre espèces (Analyse en composantes principales, mode $R$ ).

\section{Etude particulière des variations temporelles}

Le but de cette analyse consiste à rechercher les axes factoriels qui traduisent uniquement les variations dans le temps, en maintenant fixées les variations dans l'espace (on reconnait le principe de l'analyse de variance où la variation totale est décomposée en sources de variations élémentaires). Cette variante, de l'analyse précédente consiste à centrer chaque variable, non pas par la moyenne générale des observations, mais par la moyenne partielle des observations de chaque station (on procède de même pour la réduction des effectifs). Le centrage des données par "blocs" (déjà utilisé par Laurec et Le Ga11, 1974) permet d'éliminer tout décalage systématique de densité des espèces entre les trois stations et de privilégier ainsi les variations dans le temps. Effectuer séparêment une analyse d'inertie sur chacune des trois stations aboutirait à trois structures issues de trois systèmes d'axes différents qu'il serait impossible de relier les uns aux autres, 1 'analyse perdant ainsi toute unité. 


\section{Diversité}

La diversité spécifique est estimée par 1 'indice de Shannon $\left(H_{(s)}=-\sum\right.$ pi $\log _{2} p i$; $\mathrm{pi}=\frac{\mathrm{nl}}{\mathrm{N}}=$ fréquence relative de 1 'espèce $\mathrm{i}$ ) qui présente 1 'avantage de n'être subordonné à aucune hypothèse préalable sur la distribution des espèces et des individus. Afin d'obtenir une meilleure estimation des fréquences relatives de chaque espèce, et par la même, d'éviter les biais dûs à la distribution contagieuse des individus, 1 'indice de diversité est calculé, non pas sur les effectifs de chaque benne, mais sur les effectifs cumulés des bennes effectués au même endroit à la même date.

\section{RESULTATS}

\section{Analyse globale des variations spatio-temporelles}

La répartition des barycentres saisonniers (barycentres des prélèvements effectués à la même date) de chaque station (Fig. 1) dans le plan I-II de l'ana-

- lyse d'inertie met en évidence 1 'hétérogénéité de la composition faunistique entre les 3 stations. Les 3 unités de peuplement sont bien séparées selon 1 'axe I $(24,1 \%$ de la variance totale) qui représente essentiellement 1 'axe climatique. La station Mousterlin apparaît bien intermédiaire entre les deux autres. L'axe II ( $11 \%$ d'inertie) discrimine vers son pôle positif les prélèvements effectués à Mousterlin, caractérisés par des conditions écologiques instables dues à un fort hydrodynamisme. La dispersion des points dans l'espace factoriel témoigne de cette instabilité.

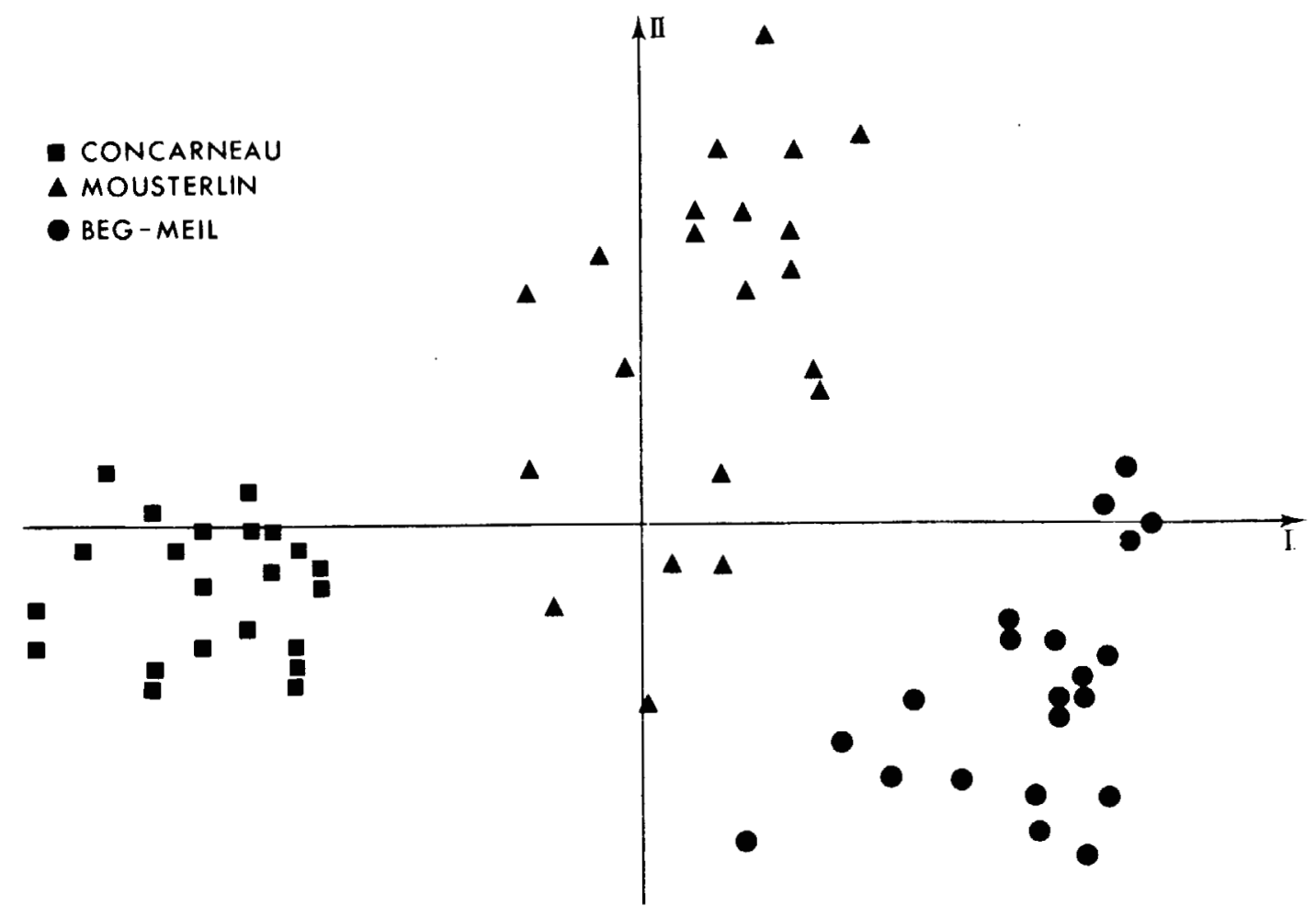

Fig. 1. Analyse globale - Répartition des prélèvements dans le plan I-II de l'analyse d'inertie - Chaque point représente le barycentre des prélèvements effectués au cours de chaque sortie à chaque station de 1970 à 1974 . 
Il n'est pas possible de repérer les cycles saisonniers au sein de chaque station. Néanmoins, la projection des barycentres annuels (Fig. 2) dans le même système d'axes I-II de 1 'analyse, suggère 1 'existence de variations pluriannuelles non négligeables. Une évolution régulière apparait de 1970 à 1972, à l'intérieur de chaque station; les projections des années 73 et 74 indiquent un retour vers une situation initiale en baie de Concarneau, tandis qu'à Beg-Meil et surtout à Mousterlin, I'évolution se poursuit vers une nouvelle composition faunistique. Les axes I et II traduisent essentiellement les effets spatiaux et la structure temporelle recherchée s'en trouve masquée. Pour lever cette hypothèque, 1 'étude des variations dans le temps est abordée par une analyse ayant les propriétés d'éliminer les variations inter-stations, pour ne considérer globalement que les variations intra-stations.

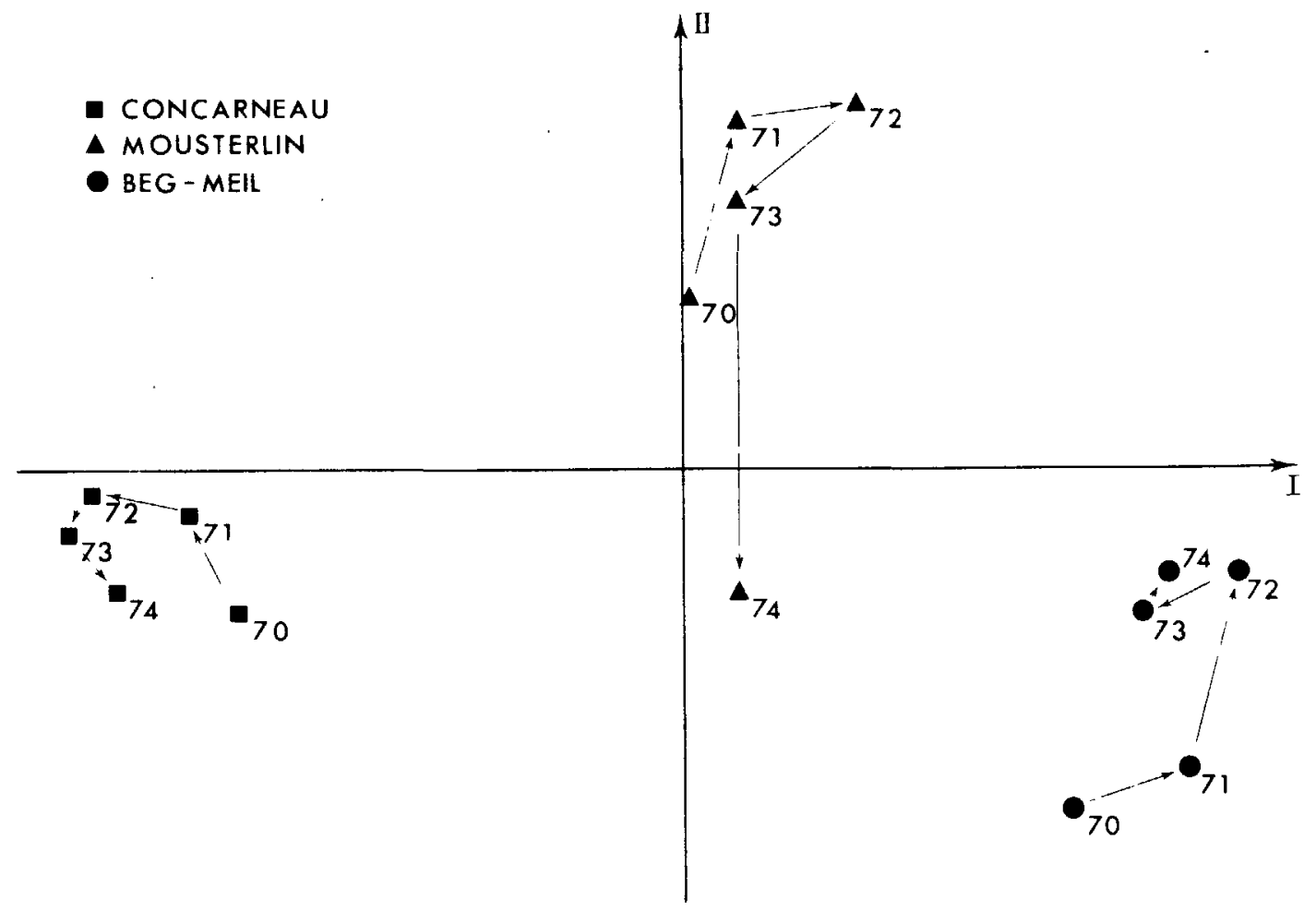

Fig. 2. Analyse globale - Répartition des barycentres des prélèvements effectués chaque année à chaque station dans le plan I-II de l'analyse d'inertie - L'espace de référence est le même que celui de la figure 1 ; les symboles également.

\section{Etude particulière des variations temporelles}

Les axes d'inertie extraits par cette analyse (Fig. 3) traduisent uniquement les variations temporelles de l'ensemble faunistique étudié. Pour simplifier la représentation graphique les barycentres sont groupés par station, pour 1970-71 et 72 d'une part, pour 73 et 74 d'autre part. On retrouve en effet, cette évolution en deux phases signalée plus haut :

- de 1970 à 1972, évolution quasi-parallèle de la composition faunistique des stations, Fig. 3, a, b, c ;

- 1973 et 1974, évolution divergente, désynchronisation des fluctuations du peuplement aux 3 stations, Fig. 3, d, e, f.

A la station baie de Concarneau, le phénomène est le plus net ; les modifications de la composition faunistique décrivent un cycle saisonnier distinct 


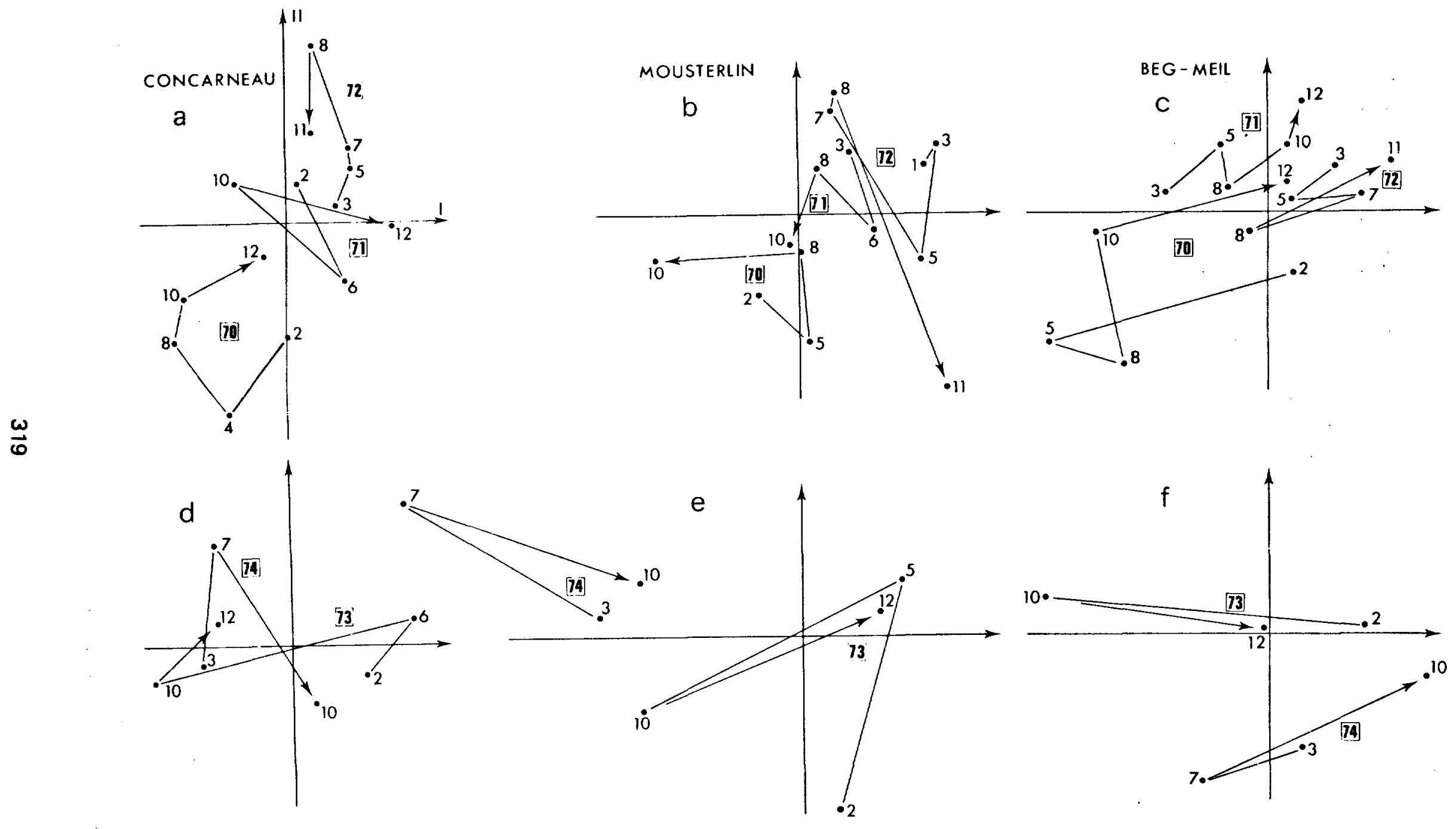

Fig. 3. Analyse particulière des variations temporelles - Analyse d'inertie sur les variables (espèces) centrées et réduites par blocs (station) - Répartition des barycentres saisonniers pour les 3 stations pendant les 5 années. 
chaque année. On constate néanmoins qu'il n'y a pas reproductibilité des variations saisonnières de chaque cycle annuel. Ces 3 cycles montrent une dérive évidente de la composition faunistique ; cette même dérive existe à Mousterlin et à Beg-Meil, tout en étant moins nette.

Durant cette période, il y a augmentation du nombre des effectifs du peuplement, enrichissement en espèces et élévation de l'indice de diversitè spécifique (Fig. 4). Cette phase apparaît comme une période d'occupation de plus en plus poussée du milieu, faisant certainement suite à des évènements survenus avant 1970. La population d'Amphiura filiformis étudiée particulièrement passe de $400 / \mathrm{m}$ à $700 / \mathrm{m}^{2}$ à la station baie de Concarneau ; de $250 / \mathrm{m}^{2}$ à $500 / \mathrm{m}^{2}$ à 1 a station Mousterlin. Parallèlement, des populations de Polychètes et de Bivalves s'accroissent régulièrement aux stations. A la station Beg-Meil, les Amphiura filiformis absentes en 1970 apparaissent ensuite sans toutefois dominer. Ce coefficient de diversité y atteint 3,5 , ce qui est très élevé.

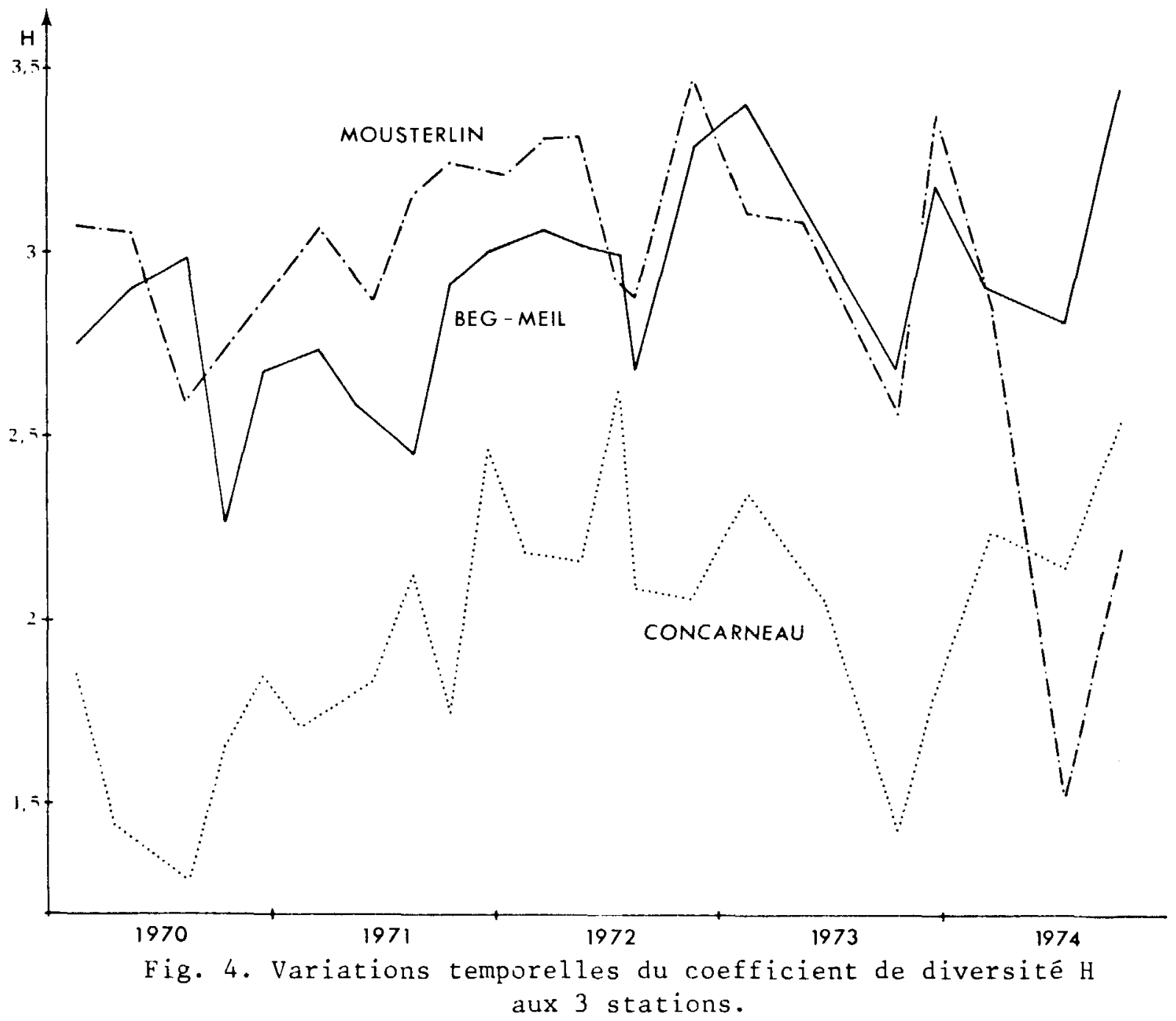

La deuxième phase de cette évolution (Fig. 3, d, e, f) montre en 1973 un retour aux conditions faunistiques rencontrées précédemment en 1970 et 1971 , ceci aux 3 stations. Inversement, en 1974, les peuplements de Mousterlin et de Beg-Meil évoluent dans une direction opposée traduisant des différences faunistiques marquées par rapport aux conditions initiales. En baie de Concarneau la situation semble se stabiliser. 
On retrouve dans l'étude des variations temporelles comme dans celles des variations spatiales, le caractère éminemment instable de la station de Mousterlin. Les fortes fluctuations de l'indice de diversité en 1973 et 74 , en cette station, confirment ce caractère.

\section{DISCUSSION}

Les phénomènes évolutifs sont plus accusés à la station baie de Concarneau, où les valeurs de l'indice de diversité sont les plus faibles; le peuplement étant dominé par un nombre très restreint d'espèces abondantes, telles qu' Amphiura filiformis, les fluctuations de la composition faunistique sont très liées à la dynamique de ces populations. Ainsi le printemps de 1973, marqué par des conditions climatiques catastrophiques, a exercé un effet négatif sur le recrutement des Amphiura filiformis et vraisemblablement sur d'autres espèces qui 1 'accompagnent dans cette période de colonisation.

Le point de rupture entre les deux phases semble lié à un phénomène climatique. Ses effets ont été d'autant plus accusés, qu'il est survenu à un moment où la compétition pour la place disponible était la plus forte. Passé ce point de rupture, les relations d'équilibre entre espèces sont modifiées, le peuplement semble désorganisé, chaque station voit son peuplement évoluer de façon particulière.

Sur 1 'ensemble de la baie de Concarneau, on note au niveau de plusieurs communautés, un envasement et un enrichissement croissant. On peut donc se demander si l'accident survenu dans l'évolution des sables envasés n'est que passager et si l'évolution avec enrichissement constant reprendra son cours. La deuxième hypothèse consiste à considêrer ce point de rupture entre les deux phases, comme tout à fait naturel, impliquant l'existence de cycles pluriannuels de l'ordre de 5 à 6 ans, appelés à se répéter. Avant de pouvoir se prononcer sur ces deux hypothèses et sur ce que peut être l'état climax au niveau de chaque peuplement, il serait souhaitable de disposer de sérias temporelles plus longues.

\section{CONCLUSION}

L'étude des fluctuations faunistiques sur 5 années au niveau d'un même ensem-ble édaphique fournit une série de données particulièrement propices à l'arai-. lyse des variations temporelles. Ces variations sont marquées par l'hétérogenéité spatiale inter-stations gouvernée principalement par le facteur climatique. L'étude particulière des variations temporelles permet de décomposer cette période de 5 années en deux phases :

- une phase à évolution faunistique parallèle aux 3 stations, phase de colonisation caractérisée par une augmentation de la densité, de la diversité et de la richesse faunistique ;

- une phase de désorganisation où les relations d'équilibre entre espèces sont modifiées et où les stations évoluent chacune de façon particulïre.

\section{REMERCIEMENTS}

Ce travail est réalisé avec l'aide financière et matérielle du Centre National pour 1'Exploitation des Océans. 


\section{REFERENCES}

Boucher, D. 1975 Production primaire saisonnière du microphytobenthos des sables envasés en baie de Concarneau.

Thèse 3è Cycle, Universitê de Brest, $113 \mathrm{p}$.

Chardy, P., Glémarec, M. \& Laurec, A. 1976 Application of inertia methods to benthic marine ecology : practical implications of the basic options. Estuar. Coastel Mar. Sc. 4, 179-205.

Glemarec, M. 1973 The benthic communities of the european north atlantic continental shelf.

Oceanogr. Mar. Biol. Ann. Rev. 11, 263-289.

Laurec, A. \& Le Gall, J.Y 1974 Application des méthodes d'analyse multivariable à l'ëtude d'une pêcherie plurispécifique : la Pêcherie Palangrière thonière en Atlantique.

Comm. Int. pour la Conservation des Thonidés de l'Atlantique, Vol. III. 